\title{
LARGE VOLUME TEM 00 MODE OPERATION OF Nd : YAG LASERS
}

\author{
D.C. HANNA, C.G. SAWYERS and M.A. YURATICH \\ Department of Electronics, University of Southampton, \\ Southampton SO9 SNH, England
}

Received 12 February 1981

\begin{abstract}
A stable resonator incorporating a suitably adjusted telescope allows operation of a NdYAG laser with a large volume $T_{E M_{00}}$ mode. The resonator design allows excellent beam quality to be reliably maintained. TEM $\mathrm{O}_{00}$ energy in excess of $350 \mathrm{~mJ}$ was obtained from a fixed $Q$ baser. $Q$-switched energies have been kept at $\sim 100 \mathrm{~mJ}$ to avoid damage but operation at $\sim 200 \mathrm{~mJ}$ appears feasible.
\end{abstract}

\section{Introduction}

There has been considerable recent work directed towards extracting as much energy as possible from a NdYAG laser in a "diffraction-limited" or "neardiffraction-limited" beam. A notable contribution was made by Herbst et al. [1] who demonstrated the capability of an unstable resonator. However a disadvantage of the usual diffraction-coupled output from an unstable resonator is the hole in the near-field intensity distribution. This proves to be an inconvenient feature for a number of applications [2] and various other approaches have been tried in order to avoid this problem. One such approach, recently reported by Sarkies [3], involves a stable resonator containing a telescope. Small changes of telescope adjustment can affect the laser beam significantly (e.g. its divergence) and Sarkies adopted an adjustment which gave a good working compromise, i.e. a large output energy but with a divergence somewhat greater than diffraction-limited. These results stimulated our own investigations of the telescopic resonator, to see whether TEM 00 mode operation could be achieved with a large volume and with a degree of reliability which made it practicable. In the course of our investigations we became acquainted with the work of Steffen, Lortscher and Herziger [4,5] on what they refer to as "dynamic stable resonators". These publications appear not to have received wide recognition, but they contain the all-important recipe for reliable operation with a large volume $\mathrm{TEM}_{00}$ mode, namely that the resonator must be designed so that the spot size in the laser rod is insensitive to fluctuations in the focal length of the pump-induced thermal lens. Various resonator designs can achieve this insensitivity and the resonator incorporating a telescope is one ${ }^{\ddagger}$. We give here a simplified discussion of the resonator design, the detailed analytical treatment to be given in a further publication [6]. From our discussion it will be apparent that the correct choice of telescope adjustment is of crucial importance (but not critical to maintain once achieved) and failure to appreciate this fact has led to some disappointing earlier results with telescopic resonators (see e.g. [1]).

\section{Resonator design}

First we consider the resonator shown in fig. 1a, with a plane mirror at one end and a spherical mirror, of curvature $f_{\mathrm{m}}$, at the other end, this being represented by a lens of focal length $f_{\mathrm{m}}$ adjacent to a plane mirror. The laser rod, represented by the (thermally induced) lens of focal length $f_{\mathrm{R}}$ is also assumed for simplicity to be adjacent to the curved mirror, and the lens combination has a focal length $f$. The beam spot size $w_{1}$ at the laser rid is given by [7],

\footnotetext{
₹ Steffen et al. [4] briefly mention this possibility but give
} no indication of having used such an arrangement. 
(a)

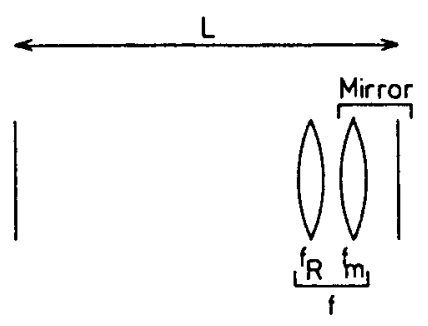

(b)

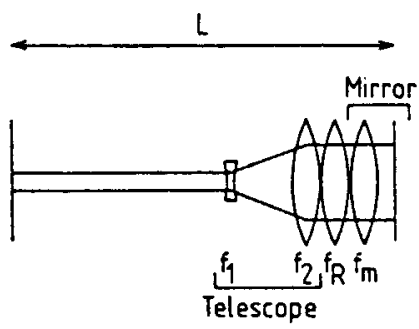

Fig. 1. a. Resonator with laser rod (focal length $f_{\mathrm{R}}$ ) close to mirror of curvature $f_{\mathrm{m}}$. The focal length, $f$, of the lens combination is given by $1 / f=1 / f_{\mathrm{R}}+1 / f_{\mathrm{m}}$. b. Resonator with laser rod (focal length $f_{\mathrm{R}}$ ) close to a plane mirror and incorporating a short telescope of magnification $M\left(f_{2}=-M F_{1}\right)$ close to the laser rod.

$w_{1}^{2}=(\lambda / \pi)[L /(f-L)]^{1 / 2}$.

Thus an arbitrarily large spot size can be obtained by making $f$ large, i.e. by arranging that $f_{\mathrm{m}} \approx-f_{\mathrm{R}}$. How. ever it can also be shown that the fractional change of spot size, $\delta w / w$, produced by a fractional change, $\delta f_{\mathrm{R}} / f_{\mathrm{R}}$, of the rod focal length is given by

$\frac{\delta w}{w}=\frac{1}{4}\left(\frac{f-2 L}{f-L}\right)\left(1+f_{\mathrm{R}} / f_{\mathrm{m}}\right)^{-1} \frac{\delta f_{\mathrm{R}}}{f_{\mathrm{R}}}$.

Thus a large spot, which requires $\left(1+f_{\mathrm{R}} / f_{\mathrm{m}}\right) \gg 1$, would generally imply large fluctuations $\delta w / w$, as a result of shot-to-shot variations of pump energy and hence of $f_{\mathrm{R}}$. This would tend to make the laser performance unreliable. However eq. (2) shows that the spot size is insensitive to fluctuations of $f_{\mathrm{R}}$ if the cavity length $L$ is chosen to satisfy the condition $f=$ $2 L$, thus giving a spot size $w_{1}=(\lambda / \pi)^{1 / 2}=(2 L \lambda / \pi)^{1 / 2}$. Steffen et al. [4] confirmed experimentally that this condition did indeed permit reliable operation with a large mode volume although the disadvantage of this design was the inconvenient resonator length implied by the large value of $f$. One way to get around this inconvenience is to use a short radius convex mirror, the approach taken by Steffen et al. [4,5]. Alternati; ly one can use a telescope arrangement as shown in fig. $1 \mathrm{~b}$, with the advantage over the convex mirror resonator of not producing such a small spot on the mirror [6]. One can best understand the role of the telescope by considering, for simplicity, a short telescope of magnification $M$ (where $f_{2}=-M f_{1}$, see fig. Ib) located close to the laser rod. Thus the lens focal lengths are taken to be short compared to the resona tor length. It can be shown that for small defocussing $\delta$ of the telescope i.e. where the lens spacing is $f_{1}+f_{2}+\delta$ and $\left|\delta / f_{1}\right|<1$, the telescope has two mai: effects: it changes the beam spot size by a factor $\sim M$ and it changes the wavefront curvature as though it consisted of a single lens of focal length $f_{\mathrm{T}}=$ $-f_{1} f_{2} M / \delta=f_{2}^{2} / \delta$. Thus the telescope can be adjusted to achieve compensation of the thermal lens $f_{\mathrm{R}}$ by making $f_{\mathrm{T}} \approx-f^{\prime}$ where $f^{\prime}$ now refers to the focal length resulting from the combination of $f_{\mathrm{R}}$ and $f_{\mathrm{m}}$, i.e. $1 / f^{\prime}=1 / f_{\mathrm{R}}+1 / f_{\mathrm{m}}$. The effect of the magnification $M$ is to modify the condition for insensitivity of spot size to variations of $f_{\mathrm{R}}$. This becomes $f / M^{2}=2 L$ where $f$ is the focal length resulting from the combin: tion of $f_{\mathrm{T}}, f_{\mathrm{R}}$ and $f_{\mathrm{m}}$ i.e. $1 / f=1 / f_{\mathrm{T}}+1 / f^{\prime}$. The spot size in the laser rod is again given by $w_{1}=(\lambda / \pi)^{1 / 2}$, which can also be written as $w_{1}=M(2 L \lambda / \pi)^{1 / 2}$. Thus introducing the correctly adjusted telescope allows the same large mode volume in the laser rod to be maintained but with a reduction of cavity length by $M^{2}$. The main limitation of this approach is that it ex poses components in the reduced beam to higher intensity and thus greater damage risk.

So far our discussion has assumed a simplified reso nator with the lenses close to one mirror, whereas in a practical resonator the optical components have a finite size and are therefore located some distance from the mirror. A more realistic treatment is then needed for accurate results and we have made exact calculations using a computer to carry out ray transfe matrix operations. In addition we have developed an approximate analysis [6] leading to simple analytical expressions for spot-size which agree very closely witt. these exact calculations. Fig. 2 shows the results of exact calculations of spot-size versus telescope defocu ing with parameters corresponding to a particular reso. nator that we have tested experimentally. The main feature to notice is the broad flat minimum for spot- 


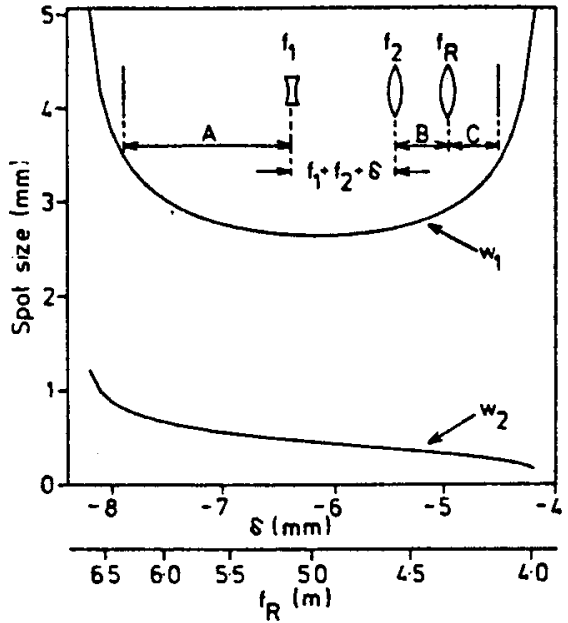

Fig. 2. Spot sizes $w_{1}$ (in laser rod) and $w_{2}$ (on left hand mirror) versus tclescope defocussing $\delta$, with $f_{\mathrm{R}}$ fixed $(5 \mathrm{~m})$, or equivalently versus $f_{R}$ with $\delta$ fixed at $-6.2 \mathrm{~mm}$. The laser parameters are as follows: $A=0.55 \mathrm{~m}, B=0.37 \mathrm{~m}, C=0.16$ $\mathrm{m}, f_{1}=-0.05 \mathrm{~m}, f_{2}=0.20 \mathrm{~m}, M=4$.

size in the laser rod (upper curve), implying insensitivity of spot-size to $\delta$. Note that a change of $\delta$ with $f_{\mathrm{R}}$ fixed is equivalent to a change of $f_{\mathrm{R}}$ (due to changed pump conditions) with $\delta$ fixed, since both correspond to a change of combined focal length $f$. Thus fig. 2 also represents a plot of spot size versus $f_{\mathrm{R}}$. The minimum of the upper curve therefore implies insensitivity to fluctuations in $f_{\mathrm{R}}$. The desired operating point is at the bottom of this minimum and the telescope must therefore be defocussed by the correct amount to ensure this. Incorrect telescope adjustment could lead to operation on the steep sides of the minimum and unreliable performance would result. Our procedure for checking the correctness of the telescope adjustment is described in the experimental section.

\section{Experimental}

In arriving at a resonator design the main parameters to be chosen are spot-size $w_{1}$ in the laser rod, resonator length $L$ and magnificiation $M$. In fact these are related and the relationship for the simplified resonator of fig. $1 \mathrm{~b}$ has already been given, viz. $w_{1}=$ $M(2 L \lambda / \pi)^{1 / 2}=(\lambda / \pi)^{1 / 2}$. Our approach has been to first choose a value of $w_{1}$ which makes good use of the available volume of laser medium. In practice the maximum value of $w_{1}$ may be limited by optical quality of the medium. the uniformity of pumping and the presence of thermally induced birefringence [8]. The laser used in our experiments was a NdYAG laser, with a rod of $75 \mathrm{~mm}$ length and $9 \mathrm{~mm}$ diameter pumped by twin close-coupled flashlamps [J.K. Lasers]. We have found it easy to operate with $w_{1}=2.5 \mathrm{~mm}$ and it is likely that even larger spot-size would be feasible. With $w_{1}$ chosen, the choice of values for $L$ and $M$ is made to give an acceptable compromise between a small $M$ and hence an inconveniently large $L$ or small $L$ and hence large $M$ which may then lead to excessive intensity in the contracted beam. When $L$ (and hence $M$ ) have been chosen the valuc of $f$ is fixed $\left(f=2 M^{2} L\right.$ for the simplified resonator) and this in turn fixes $\delta$ through the relations $1 / f=1 / f_{\mathrm{T}}+1 / f_{\mathrm{R}}$ $+1 / f_{\mathrm{m}}$ and $f_{\mathrm{T}}=f_{2}^{2} / \delta$ ). This assumes $f_{\mathrm{R}}$ to be known - we have measured $f_{\mathrm{R}}$ for various lamp powers $P$ and found that the relation $f_{\mathrm{R}}(m)=2.7 / P(\mathrm{~kW})$ is obeyed [see 8]. With the design value of $\delta$ fixed, the telescope is first adjusted to be exactly collimated (using an autocollimator), then a calculated correction is made for dispersion so that it is collimated for 1.06 $\mu \mathrm{m}$, and finally the design value of $\delta$ is introduced. With the telescope so adjusted further adjustments are made to the laser as follows. (i) the mirror alignment is adjusted for maximum output energy. (ii) $A$ circular aperture to select the $\mathrm{TEM}_{00}$ mode is inserted and centred. In practice we have found that the aperture diameter should be $\sim 1.5$ times the calculated spot diameter at the point of insertion to ensure suppression of the TEM 01 mode. For convenience the aperture is used in the expanded beam. (iii) Fine adjustment of $\delta$ is made until the beam circularity and uniformity, as seen from burn patterns on photographic paper have been optimised. Measurements of beam intensity profile and beam divergence have then been made using a Si photodiode array. Observations have been made for a number of resonator mirror combinations, at various pump levels, both on the expanded and contracted beams and for fixed- $Q$ and $Q$-switched operation. It has been found under these various conditions that by adopting the alignment procedure outlined above an excellent beam quality can be achieved and reliably maintained, with the measured profile and divergence agreeing to within experimental accuracy with values calculated from the design equations. A 
typical resonator design that we have used is specified by the parameters given in fig. 2 . With this resonator and with fixed- $Q$ operation, a TEM 00 output energy of $350 \mathrm{~mJ}$ has been obtained for a flashlamp input energy of $55 \mathrm{~J}$. When $Q$-switched we have operated at lower energy to avoid damage to the small lens of the telescope and to the reflector for the contracted beam. $A$ resonant reflector (Spectrosil $B$ ) and uncoated lens (BK7) have been used, taking the contracted beam as output. At first, using a lens whose surface finish was suspect, is was found that damage to the lens was sustained at $150 \mathrm{~mJ}$ output whereas the resonant reflector was unscathed. With a replacement lens (aiso BK7), the output has been deliberately restricted to $\sim 100$ $\mathrm{mJ}$ and no sign of damage found after more than $10^{5}$ shots. As a test of the laser's reliability we have run it at the $\sim 100 \mathrm{~mJ}$ level continuously for one hour at 8 $\mathrm{Hz}$ without any adjustment. No special efforts were made to stabilise the laser mechanically or thermally. During this time the burn patterns at a distance of several metres from the output mirror were examined periodically. The burn patterns remained visually identical, with perfect circularity and complete freedom from any structure, diffraction rings, etc. We have found that $\mathrm{TEM}_{01}$ mode operation can be induced by small displacements of the aperture off-axis (the two lobes then have quite different intensities); even a small admixture of $\mathrm{TEM}_{01}$ mode is then visually detectable in the burn patterns. No sign of $\mathrm{TEM}_{01}$ operation was found during the one hour trial.

In conclusion we have obtained reliable operation of a NdYAG laser with a large volume $\mathrm{TEM}_{00}$ mode of excellent purity. A design procedure for achieving this by using a telescopic resonator has been outlined. A change of pumping conditions, e.g. repetition rate or input energy, only requires a (readily calculable) ad. justment of telescope spacing. $Q$-switched output ener. gies have been limited to $100 \mathrm{~mJ}$ in our experiments but no attempt has been made to optimise the design as far as damage threshold is concerned. With optimisation and by paying attention to the choice and protection of the vulnerable components (e.g. by eliminat. ing dust) it is believed that $Q$-switched $\mathrm{TEM}_{00}$ outputs at $\sim 200 \mathrm{~mJ}$ would be a practical possibility.

This work has been supported by a grant from the Science Research Council and one of us (C.G.S.) wishes to acknowledge support from the S.R.C. and UKAEA (Culham Laboratory) in the form of a Case Studentship. We also wish to acknowledge helpful discussions with Dr.P.H. Sarkies and assistance in the latter phase of the work from Mr. A.J. Berry.

\section{References}

[1] R.L. Herbst, H. Komine and R.L. Byer, Optics Comm. 21 (1977) 5.

[2] D.C. Hanna and L.C. Laycock, Opt. Quant. Elect. 11 (1979) 153.

[3] P.H. Sarkies, Optics Comm. 31 (1979) 189.

[4] J. Steffen, J.P. Lörtscher and G. Herziger, IEEE J. Quant. Elec. QE-8 (1972) 239.

[5] J.P. Lörtscher, J. Steffen and G. Herziger, Opt. Quant. Elect. 7 (1975) 505.

[6] D.C. Hanna, C.G. Sawyers and M.A. Yuratich, (in preparation).

[7] H. Kogelnik and T. Li, Appl. Optics S (1966) 1550.

[8] W. Koechner, Solid state laser engineering, Vol. 1, Springer Series in Optical Sciences (Springer-Verlag, 1976). 